\title{
Positive and Negative Particle Masses in the Bicubic Equation Limiting Particle Velocity Formalism
}

\author{
Josip Śoln ${ }^{1}$ \\ ${ }^{1}$ JZS Phys-Tech, Vienna, Virginia 22182, USA \\ Correspondence: Josip Śoln, JZS Phys-Tech, Vienna, Virginia 22182, USA. E-mail: jurasoln@yahoo.com \\ Received: December 6, 2017 \\ Accepted: December 20, 2017 \\ Online Published: January 30, 2018 \\ doi:10.5539/apr.v10n1p14 \\ URL: https://doi.org/10.5539/apr.v10n1p14
}

\begin{abstract}
The interest in the negative particle mass here got encouraged by the Rachel Gaal July 2017 APS article (Gaal, 2017) describing Khamehchi et al. (2007) observation of an effective negative mass in a spin-orbit coupled Bose-Einstein condensate. Hence, since in the bicubic equation limiting particle velocity formalism (Śoln, 2014, 2015, 2016, 2017) positive $m_{+}=m>0$ and negative $m_{-}=-m<0$ masses with $m_{+}^{2}=m_{-}^{2}=m^{2}$ are equally acceptable, then from a purely theoretical point of view, the evaluation of particle limiting velocities for both $m_{+}$and a $m_{-}$masses should be done.

Starting with the original solutions for particle limiting velocities $c_{1}, c_{2}$ and $c_{3}$, given basically for a positive particle mass $m_{+}$(Śoln, 2014, 2015, 2016, 2017), now also are done for a negative particle mass $m_{-}$This is done consistent with the bicubic equation mathematics, by solving for $c_{1}, c_{2}$ and $c_{3}$ not only for $m_{+}$but also for $m_{-}$. Hence, in addition to having the limiting velocities of positive mass $m_{+}$primary, obscure and normal particles, now one has also the limiting velocities of negative mass $m_{-}$primary, obscure and normal particles, however, numerically equal to limiting velocities, respectively of $m_{+}$masses obscure, primary and normal particles, forming the $m_{+}$and $m_{-}$masses of equal limiting velocity value doublets : $c_{1}(m-)=c_{2}\left(m_{+}\right), c_{2}\left(m_{-}\right)=c_{1}\left(m_{+}\right), c_{3}\left(m_{-}\right)=c_{3}\left(m_{+}\right)$. Now, one would like to know as to which particle with a negative mass $m_{-}=-m \prec 0$, obtained from the positive mass $m_{+}=m>0$ with the substitution $m \longrightarrow-m$, can have a real limiting velocity? It turns out that it is the obscure particle limiting velocity $c_{2}\left(m_{+}\right)$that changes from the imaginary value, $c_{2}^{2}\left(m_{+}\right) \prec 0$, into the real limiting velocity value $c_{2}^{2}\left(m_{-}\right)>0$ when the change $m_{+} \longrightarrow m_{-}$is made and, at the same time, retaining the same energy. Similar procedure applied to the original primary particle limiting velocity starting with

$c_{1}^{2}\left(m_{+}\right)>0$, keeping the total energy the same, with the change $m \longrightarrow-m$ one ends up with $c_{1}^{2}\left(m_{-}\right)<0$ that is, imaginary $c_{1}$. The procedure of changing $m_{+} \longrightarrow m_{-}$in normal particle limiting velocity causes no change, it remains the same real $c_{3}$. Because $m^{2}\left(=m_{+}^{2}=m_{-}^{2}\right), E^{2}$ and $v^{2}$ remain the same, these mass regenerations, $m_{+} \longrightarrow m_{-}$and $m_{-} \longrightarrow m_{+}$could in principle also occur spontaneously.
\end{abstract}

Keywords: add 3 to 5 keywords

\section{Introduction}

The beauty of the bicubic equation solutions for the primary, obscure and normal particle respective limiting velocities $c_{1}, c_{2}$ and $c_{3}$ (Śoln, 2014, 2015, 2016, 2017) with implicitely assuming $m>0$ is in the fact that the congruent parameter $z=3 \sqrt{3} m v^{2} / 2 E$ with $m, v$ and $E$ particle mass, velocity and energy is not only evolutionary but also restrictive on $c_{1}$, $c_{2}$ and $c_{3}$ due to the condition $-1 \leq z \leq 1$ (Śoln, 2014, 2015, 2016, 2017). Actually, as $m, v^{2}$ and $E$ are practically always positive the condition on the congruent parameter is basically only $0 \leq z \leq 1$. By introducing a negative mass, $m_{-}=-m \prec 0$, and with $v \geq 0$ and $E \geq 0$, one is dealing now with the new additional range for $z,-1 \leq z \leq 0$.

The announcement by Gaal (2017) of the effective negative mass observation by Khamehchi et al. (2017) in a spin-orbit coupled Bose -Einstein condensate immediately raises a question, is this an isolated case or is it generally allowed, say by the bicubic equation limiting particle velocity formalism (Śoln, 2014, 2015, 2016, 2017).

Here, rather than get involved into the complexity as to why and how the negative mass $m_{-} \prec 0$ occurred in Khamehchi et al. (2017) and more recently in Li \& Cui (2017) and Dold (2017), one would like to see how the expressions for limiting velocities $c_{1}, c_{2}$ and $c_{3}$ change when an explicitly negative mass $m_{-}=-m \leqslant 0$ is introduced. Working with $|z|<<1$, one finds specifically that the obscure particle, when one changes $m_{+} \rightarrow-m<0$, assumes the primary particle form with positive rest energy and positive kinetic energy, with its $c_{2}$ becoming real, $c_{2}^{2}>0$. At the same time, the primary particle 
originally with $m_{+}=m>0$ and $c_{1}^{2}>0$ and positive rest and kinetic energies, with the change $m_{+}=m \rightarrow m_{-}=-m \leqslant 0$, assumes the obscure particle form with now $c_{1}^{2}$ becoming negative with positive rest energy but negative kinetic energy. Because of the small congruent parameter values, $|z| \prec<1$, with $m_{+} \rightarrow-m, m>0$, the normal particle only implicitly indicates that it still has positive rest energy and positive kinetic energy with $c_{3}$ remaining real. In fact, what one has here is that one has positive to negative mass regeneration: primary to obscure, obscure to primary and normal to normal, as well as negative to positive mass regeneration: primary to obscure, obscure to primary and normal to normal.

What is very important to keep in mind, as established in Śln $(2014,2015,2016,2017)$, is the fact that for different limiting velocity solutions $c_{1}, c_{2}$ and $c_{3}, m^{2}, v^{2}$ and $E^{2}$ retain the same numerical values, even when in a variety of convenient functional forms with $c_{1}, c_{2}$ and $c_{3}$.

In Section 2 the bicubic equation limiting velocity solutions $c_{1}, c_{2}$ and $c_{3}$ are separated by the positive and negative $m$, respectively, $m_{+}=m>0, m_{-}=-m<0$. The detailed evaluation establishes that the $m_{+}$and $m_{-}$masses form the equal value limiting velocity doublets : $c_{1}\left(m_{-}\right)=c_{2}\left(m_{+}\right), c_{2}\left(m_{-}\right)=c_{1}\left(m_{+}\right), c_{3}\left(m_{-}\right)=c_{3}\left(m_{+}\right)$.

Section 3 is devoted to the physical difference of each of the limiting velocities $c_{1}, c_{2}$ and $c_{3}$ between the positive, $m_{+}$, and negative $m_{-}$mass. Here also the possibility of a negative mass of $m_{-}=-m$ with $m=\left|m_{-}\right| \geq 100 \mathrm{GeV}$ is discussed as a possible dark matter particle in the Milky Way as opposite to already assumed $m_{+}=m \geq 100 \mathrm{GeV}$ from (Laha, 2016; Śln, 2017).

Final remarks and conclusion are given in Section 4. Here it is pointed out that the interrelationships between the limiting velocity solutions of particles with positive and negative masses may be opening a new physics avenue.

\section{Correlations between positive and negative particle masses regenerated in the bicubic equation limiting velocity solutions}

Denoting generic limiting velocity by $c$, following references (Śoln, 2014, 2015, 2016, 2017) one ends up for $c$ with the bicubic equation

$$
m^{2}\left(c^{2}\right)^{3}-E^{2} c^{2}+E^{2} v^{2}=0
$$

where $m, E$ and $v$ are particle mass, energy and velocity. In writing down the solutions for (1.1), one uses the general expression for the discriminant through the general expression of the congruent parameter $z$ (Śoln, 2014, 2015, 2016, 2017),

$$
\begin{aligned}
& z(m)=\frac{3 \sqrt{3} m v^{2}}{2 E} ;-1 \leq z(m) \leq 1, \\
& D=\left(\frac{27}{8}\right)^{2} \frac{1}{z(m)^{4}}\left(1-\frac{1}{z(m)^{2}}\right)<0
\end{aligned}
$$

Since by and large the mass $m$ so far has been always positive, here, in order to distinguish two opposite in sign masses, one uses $m_{+}$and $m_{-}$notations together with specific designations $m_{+}=m>0$ and $m_{-}=-m<0$. With these one writes the solutions of (1.1) according to Soln $(2014,2015,2016,2017)$ for respectively, primary, obscure and normal limiting velocities $\left(c_{1}, c_{2}\right.$ and $\left.c_{3}\right)$ with $m_{+}$masses as follows,

$$
\begin{gathered}
m_{+}=m \geq 0,0 \leq z\left(m_{+}\right) \leq 1: 0 \leq z(m) \leq 1 \\
\frac{c_{1}^{2}\left(m_{+}\right)}{v^{2}}=\frac{3}{z(m)} \sin \left[\frac{1}{3}\left(\pi-\sin ^{-1}(z(m))\right)\right]>0, \\
\frac{c_{2}^{2}\left(m_{+}\right)}{v^{2}}=-\frac{3}{z(m)} \sin \left[\frac{1}{3}\left(\pi+\sin ^{-1}(z(m))\right)\right] \prec 0, \\
\frac{c_{3}^{2}\left(m_{+}\right)}{v^{2}}=\frac{3}{z(m)} \sin \left[\frac{1}{3} \sin ^{-1}(z(m))\right]>0
\end{gathered}
$$

A very important property of the bicubic equation for the particle limiting velocities (1.1) is the fact that it is invariant under the substitution $m \longrightarrow-m$ which, with the fact tat $(-m)^{2}=m^{2}$, preserves the numerical values of $m^{2}, v^{2}$ and $E^{2}$, but perhaps in different functional forms with respective $c_{1}, c_{2}$ or $c_{3}$. Exploiting this invariance, the limiting velocity solutions of $m_{+}=m \geq 0$ can be easily compared with the ones obtained with changed $m \longrightarrow-m=m_{-}$for respectively, primary, obscure and normal limiting velocities $\left(c_{1}, c_{2}\right.$ and $\left.c_{3}\right)$ with $m_{-}$masses as follows 


$$
\begin{gathered}
m_{-}=-m_{+}=-m, z\left(m_{-}\right)=-z\left(m_{+}\right),-1 \leq z\left(m_{-}\right) \leq 0,0 \leq z(m) \leq 1 ; \\
\frac{c_{1}^{2}\left(m_{-}\right)}{v^{2}}=\frac{3}{z\left(m_{-}\right)} \sin \left[\frac{1}{3}\left(\pi-\sin ^{-1}\left(z\left(m_{-}\right)\right)\right)\right]=-\frac{3}{z(m)} \sin \left[\frac{1}{3}\left(\pi+\sin ^{-1}(z(m))\right)\right]<0, \\
\frac{c_{2}^{2}\left(m_{-}\right)}{v^{2}}=-\frac{3}{z\left(m_{-}\right)} \sin \left[\frac{1}{3}\left(\pi+\sin ^{-1}\left(z\left(m_{-}\right)\right)\right)\right]=\frac{3}{z(m)} \sin \left[\frac{1}{3}\left(\pi-\sin ^{-1}(z(m))\right)\right]>0, \\
\frac{c_{3}^{2}\left(m_{-}\right)}{v^{2}}=\frac{3}{z\left(m_{-}\right)} \sin \left[\frac{1}{3} \sin ^{-1}\left(z\left(m_{-}\right)\right)\right]=\frac{3}{z(m)} \sin \left[\frac{1}{3} \sin ^{-1}(z(m))\right]>0
\end{gathered}
$$

In arriving to solutions with $m_{-}=-m<0$, one took into account that $\sin ^{-1}(-x)=-\sin ^{-1}(x)$. in the $-\pi / 2$ to $\pi / 2$ range. One notices that the squares of primary and obscure limiting limiting velocities exchanged the forms when going from (2) to (3): The primary $c_{1}^{2}\left(m_{-}\right)(3.2)$ is in the old (2.2) obscure form while the obscure $\left.c_{2}^{2} m_{-}\right)(3.3)$ is in the old primary (2.2) form and the square of normal limiting velocity $c_{3}^{2} m_{-}$) (3.4) retained the same form (2.4). The retention of similar forms when going from solutions (2) to (3) is not surprising since the parameters $m^{2}, v^{2}$ and $E^{2}$ remained the same when going from (2) to (3).

Although we are talking about limiting velocities with positive $m_{+}$masses and $m_{-}$masses it is the single mass parameter $m$ that enters into the respective limiting velocity solutions (2) and (3). This makes it easier to identify numerically equal limiting velocities which also carry the same $E, m^{2}$ and $v$. From these equalities one can see, for example, how, say, the presumed spontaneous disappearance of $m_{+}$with the primary $c_{1}^{2}\left(m_{+}\right)$might regenerate $m_{-}$together with the obscure $c_{2}^{2}\left(m_{-}\right)$of equal value to primary $c_{1}^{2}\left(m_{+}\right)$. Of course, one can reverse the process and assume that the disappearance of $m_{-}$with he obscure $c_{2}^{2}\left(m_{-}\right)$might regenerate $m_{+}$with the primary $c_{1}^{2}\left(m_{+}\right)$of equal value to the obscure $c_{2}^{2}\left(m_{-}\right)$. To get a more complete picture one sets up the table of equal limiting velocities,deduced from relations (2) and (3).

Table 1. Equal values of squares of limiting velocities with $\mathbf{m}_{+}$and $m_{-}$masses with equal values of $E, m^{2}$ and $v^{2}$ and with which one might regenerate each other

$$
\begin{aligned}
& \frac{c_{1}^{2}\left(m_{+}\right)}{v^{2}}=\frac{c_{2}^{2}\left(m_{-}\right)}{v^{2}} \quad((2.2) ;(3.3)) \\
& \frac{c_{2}^{2}\left(m_{+}\right)}{v^{2}}=\frac{c_{1}^{2}\left(m_{-}\right)}{v^{2}} \quad((2.3) ;(3.2)) \\
& \frac{c_{3}^{2}\left(m_{+}\right)}{v^{2}}=\frac{c_{3}^{2}\left(m_{-}\right)}{v^{2}} \quad((2.4) ;(3.4))
\end{aligned}
$$

From expressions for the congruent parameter in relations (2) and (3), one can write the corresponding energies for each of the limiting velocities as $E=3 \sqrt{3} m_{+,-} v^{2} / 2 z\left(m_{+,-}\right)$by evaluating

$v^{2} / 2 z\left(m_{+,-}\right)$from relations (2) and (3) to obtain different expressions for $E$ but with the same numerical value (Śoln, 2016, 2017).

$$
\begin{gathered}
m_{+}=m=-m_{-}, 0 \leq z\left(m_{+}\right) \leq 1 ;-1 \leq z\left(m_{-}\right) \leq 0, \\
c_{1}^{2}\left(m_{+}\right): E=\frac{\sqrt{3} m c_{1}^{2}\left(m_{+}\right)}{2 \sin \left[\frac{1}{3}\left(\pi-\sin ^{-1}(z(m))\right)\right]}=m c_{1}^{2}\left(m_{+}\right)\left(1-\frac{v^{2}}{c_{1}^{2}\left(m_{+}\right)}\right)^{-\frac{1}{2}}, \\
c_{2}^{2}\left(m_{+}\right): E=\frac{\sqrt{3} m\left(-c_{2}^{2}\left(m_{+}\right)\right)}{2 \sin \left[\frac{1}{3}\left(\pi+\sin ^{-1}(z(m))\right]\right.}=m\left(-c_{2}^{2}\left(m_{+}\right)\right)\left(1+\frac{v^{2}}{\left(-c_{2}^{2}\left(m_{+}\right)\right)}\right)^{-\frac{1}{2}}, \\
c_{3}^{2}\left(m_{+}\right): E=\frac{\sqrt{3} m c_{3}^{2}\left(m_{+}\right)}{2 \sin \left[\frac{1}{3} \sin ^{-1} z(m)\right]}:=m c_{3}^{2}\left(m_{+}\right)\left(1-\frac{v^{2}}{c_{3}^{2}\left(m_{+}\right)}\right)^{-\frac{1}{2}} ; \\
c_{1}^{2}\left(m_{-}\right): E=\frac{\sqrt{3} m\left(-c_{1}^{2}\left(m_{-}\right)\right)}{2 \sin \left[\frac{1}{3}\left(\pi+\sin ^{-1}(z(m))\right]\right.}=m\left(-c_{1}^{2}\left(m_{-}\right)\right)\left(1+\frac{v^{2}}{\left(-c_{1}^{2}\left(m_{-}\right)\right)}\right)^{-\frac{1}{2}},
\end{gathered}
$$




$$
\begin{gathered}
c_{2}^{2}\left(m_{-}\right): E=\frac{\sqrt{3} m c_{2}^{2}\left(m_{-}\right)}{2 \sin \left[\frac{1}{3}\left(\pi-\sin ^{-1}(z(m))\right)\right]}=m c_{2}^{2}\left(m_{-}\right)\left(1-\frac{v^{2}}{c_{2}^{2}\left(m_{-}\right)}\right)^{-\frac{1}{2}}, \\
c_{3}^{2}\left(m_{-}\right): E=\frac{\sqrt{3} m c_{3}^{2}\left(m_{-}\right)}{2 \sin \left[\frac{1}{3} \sin ^{-1} z(m)\right]}:=m c_{3}^{2}\left(m_{-}\right)\left(1-\frac{v^{2}}{c_{3}^{2}\left(m_{-}\right)}\right)^{-\frac{1}{2}}
\end{gathered}
$$

\section{Possible physics with a negative particle mass. Negative mass obscure particle with a positive limiting velocity,as a candidate for a dark matter particle in the Milky Way}

The simplest way to see the effect of a negative mass on the limiting velocities is to express the limiting velocities from relations (2) and (3) as Taylor series in terms of common congruent parameter $z(m)(z(-m)=-z(m))$. Hence, similarly, as in Śln $(2016,2017)$ here one writes the Taylor series with common parameters $m^{2}, v^{2}$ and $E^{2}$ first for limiting velocities $c_{i}^{2}\left(m_{+}\right)$and then for $c_{i}^{2}\left(m_{-}\right), i=1.2,3$, respectively from relations (2), (3), (4) and (5) in terms of $z(m) \ll 1$. Starting with positive mass $m_{+}=m$, from relations (2) one obtains,

$$
\begin{gathered}
m_{+}=m \geq 0, z\left(m_{+}\right)=z(m), 0 \leq z(m) \leq 1 ; \\
\frac{c_{1}^{2}\left(m_{+}\right)}{v^{2}}=\frac{3 \sqrt{3}}{2 z(m)}-\frac{1}{2}+O(z(m))>0: E \simeq m c_{1}^{2}\left(m_{+}\right)+\frac{m v^{2}}{2}-m v^{2} O(z(m)) ; \\
\frac{c_{2}^{2}\left(m_{+}\right)}{v^{2}}=-\frac{3 \sqrt{3}}{2 z(m)}-\frac{1}{2}+O(z(m))<0: E \simeq m\left(-c_{2}^{2}\left(m_{+}\right)\right)-\frac{m v^{2}}{2}+m v^{2} O(z(m)) ; \\
\frac{c_{3}^{2}\left(m_{+}\right)}{v^{2}}=1+O(z(m))>0: E=E .
\end{gathered}
$$

Similarly, for the negative mass $m_{-}=-m \prec 0$, the Taylor series in the congruent parameter $z\left(m_{-}\right)=-z(m),|z(-m)| \ll 1$, applied to solutions (3) yield, with writing $m$ for $\left|m_{-}\right|$

$$
\begin{gathered}
m_{-}=-m \leqslant 0, z\left(m_{-}\right)=-z(m),-1 \leq z\left(m_{-}\right) \leq 0,0 \leq z(m) \leq 1 \\
\frac{c_{1}^{2}\left(m_{-}\right)}{v^{2}}=-\frac{3 \sqrt{3}}{2 z(m)}-\frac{1}{2}+O(z(m))<0: E=m\left(-c_{1}^{2}\left(m_{-}\right)\right)-\frac{m v^{2}}{2}+m v^{2} O(z(m)) \\
\frac{c_{2}^{2}\left(m_{-}\right)}{v^{2}}=\frac{3 \sqrt{3}}{2 z(m)}-\frac{1}{2}+O(z(m))>0: E=m c_{2}^{2}\left(m_{-}\right)+\frac{m v^{2}}{2}-m v^{2} O(z(m)) \\
\frac{c_{3}^{2}\left(m_{-}\right)}{v^{2}}=1+O(z(m))>0: E=E .
\end{gathered}
$$

The zero sum rule for squares of limiting velocities are satisfied not only for exact expressions in $(2.1,2,3,4)$ and $(3$. 1 , $2,3,4)$ but also here, to a very good approximation, for perturbation expressions $(6.1,2,3,4)$ and $(7.1,23,4)$,

$$
\sum_{i=1}^{4} c_{i}^{2}\left(m_{+}\right)=0, \sum_{i=1}^{4} c_{i}^{2}\left(m_{-}\right)=0
$$

In fact, in the approximation to $O(z(m))$ with $z(m) \ll 1$, the relations $(8,1,2)$ determine respectively that $c_{3}^{2}\left(m_{+}\right) \simeq v^{2}$ and $c_{3}^{2}\left(m_{-}\right) \simeq v^{2}$ that is that the normal limiting velocities are the same for $m_{+}=m$ and for $m_{-}=-m$. The higher order terms, however, may bring out the difference.

At this point, as an example of a possible negative mass obscure dark matter particle in the Milky Way (Khamehchi et al., 2017), one chooses from the characteristic dark matter Milky Way Dark Matter Velocity Profile (Khamehchi et al., 2017; Śln, 2017) the value of $v=(5 / 6) \cdot 10^{-3} c$. With this characteristic velocity, one approximates $z\left(m_{-}\right)=-z(m)$ as follows (Śoln, 2017) $\left(m=-m_{-} ;-1 \leq z\left(m_{-}\right) \leq 0\right)$

$$
\begin{gathered}
z\left(m_{-}\right)=\frac{3 \sqrt{3} m_{-} v^{2}}{2 E}=\frac{3 \sqrt{3} v^{2}}{2 c^{2}} \frac{m_{-} c^{2}}{E}=1.8 \cdot 10^{-6} \cdot \frac{m_{-} c^{2}}{E} ; \\
\frac{m_{-} c^{2}}{E} \succcurlyeq-1: z\left(m_{-}\right) \gtrsim-\frac{3 \sqrt{3} v^{2}}{2 c^{2}} \simeq-1.8 \cdot 10^{-6} ; z(m) \lesssim 1.8 \cdot 10^{-6} ;
\end{gathered}
$$




$$
(7.3): \frac{c_{2}^{2}\left(m_{-}\right)}{v^{2}} \gtrsim 1.44 \cdot 10^{6}: c_{2}^{2}\left(m_{-}\right) \gtrsim c^{2}, E \gtrsim-m_{-} c^{2}-m_{-} c^{2} O\left(10^{-6}\right) .
$$

where from relations (7.3) one estimated $c_{2}^{2}\left(m_{-}\right)$together with $E$.

The interesting thing is that the negative mass particle in this example can have a limiting velocity comparable to $c$ and that the kinetic energy is small as compared to the rest energy $-m_{-} c^{2}$, which, however is irrelevant. Now, for a ordinary dark matter mass $m$, Laha (2016) posts possibilities of values of $m c^{2} \geq 100 \mathrm{GeV}$. One may entertain the idea that a similar thing may exist also for a negative mass, expressed with its absolute value, $m c^{2}=\left|m_{-}\right| c^{2} \geq 100 \mathrm{GeV}$, in which case the negative mass kinetic energy in (9.3) could be in the $\mathrm{keV}$ range, $\left|m_{-}\right| c^{2} 0^{-6} \gtrsim \mathrm{keV}$.

\section{Discussion and conclusion}

Already in general limiting velocity expressions (2) and (3), one notices that changing the mass $m_{+}=m \geq 0$ to a mass $m_{-}=-m \leq 0$, one basically exchanges the forms of primary with obscure limiting velocity while the normal form remaining the same. The opposite exchange of the forms of obscure with primary limiting velocity happens when changing $m_{-}=-m$ to $m_{+}=m$ and again the normal forms being the same. Now, in these procedures numerically $E, v^{2}$ and $m^{2}$ do not change $\left(m_{+}^{2}=m_{-}^{2}=m^{2}\right)$ but their interrelation expressions (4) and (5) differ explicitly. Never the less, the energy expression with negative mass obscure particle (5.3) is numerically equal to the energy expression of positive mass obscure particle (4.3).

The obscure particle shows the most important change when the positive mass, $m_{+}=m>0$, is changed to the negative mass, $m_{-}=-m<0$, resulting in replacement of the positive mass unobservable imaginary limiting velocity $c_{2}\left(m_{+}\right)$ $\left(c_{2}^{2}\left(m_{+}\right)<0\right)$ with the negative mass real limiting velocity $c_{2}\left(m_{-}\right)\left(c_{2}^{2}\left(m_{-}\right)>0\right)$, which in principle could be observable. This, possibly could be one of the factors in observation of the effective negative mass in a spin-orbit coupled Bose Einstein condensate by Khamehchi et al. (2017) and popularized by Gaal (2017). The example of negative mass obscure particle also yields reasonable values of limiting velocity and the energy when considered as a dark matter particle in the Milky Way (Khamehchi et al., 2017; Śoln, 2017).

More recently, the repulsive Fermi polarons with negative effective mass have been reported from China by Li and Cui (2017); while, from the Great Britain, in the four-dimensional Riemannian Eguchi-Hanson space the negative mass has been reported by Dold (2017). It appears that the negative mass may be finally presenting itself.

\section{References}

Dold, D. (2017). Global dynamics of asymptotically locally AdS spacetimes with negative mass. arXiv preprint arXiv:1711.06700.

Gaal, R. (2017). To disperse or not to disperse: Debating negative mass. APS News, 26(7), 1.

Khamehchi, M. A., Hossain, K., Mossman, M. E., Zhang, Y., Busch, T., Forbes, M. M., \& Engels, P. (2017). NegativeMass Hydrodynamics in a Spin-OrbitCcoupled Bose-Einstein Condensate. Physical Review Letters, 118(15), 155301.

Laha, R. (2016). The effect of dark matter velocity profile on directional detection of dark matter. arXiv preprint arXiv: 1610.08632 .

Li, W., \& Cui, X. (2017). Repulsive Fermi polarons with negative effective mass. Physical Review A, 96(5), 053609.

Śoln, J. (2014). Theoretical particle limiting velocity from the bicubic equation: Neutrino example. Physics Essays, $27(3), 448-453$.

Śoln, J. (2015). Soln, J. (2015). Particle limiting velocities from the bicubic equation derived from Einsteins kinematics: PeV electron case. Applied Physics Research, 7(4), 37.

Śoln, J. (2016). Limiting Velocities of Primary, Obscure and Normal Particles: Self-Annihilating Obscure Particle as an Example of Dark Matter Particle. Applied Physics Research, 8(5), 1.

Śoln, J. (2017). Connecting dark matter particles with the primary, obscure and normal particles through implicit causality. Applied Physics Research, 9(3), 1.

\section{Copyrights}

Copyright for this article is retained by the author(s), with first publication rights granted to the journal.

This is an open-access article distributed under the terms and conditions of the Creative Commons Attribution license (http://creativecommons.org/licenses/by/4.0/). 